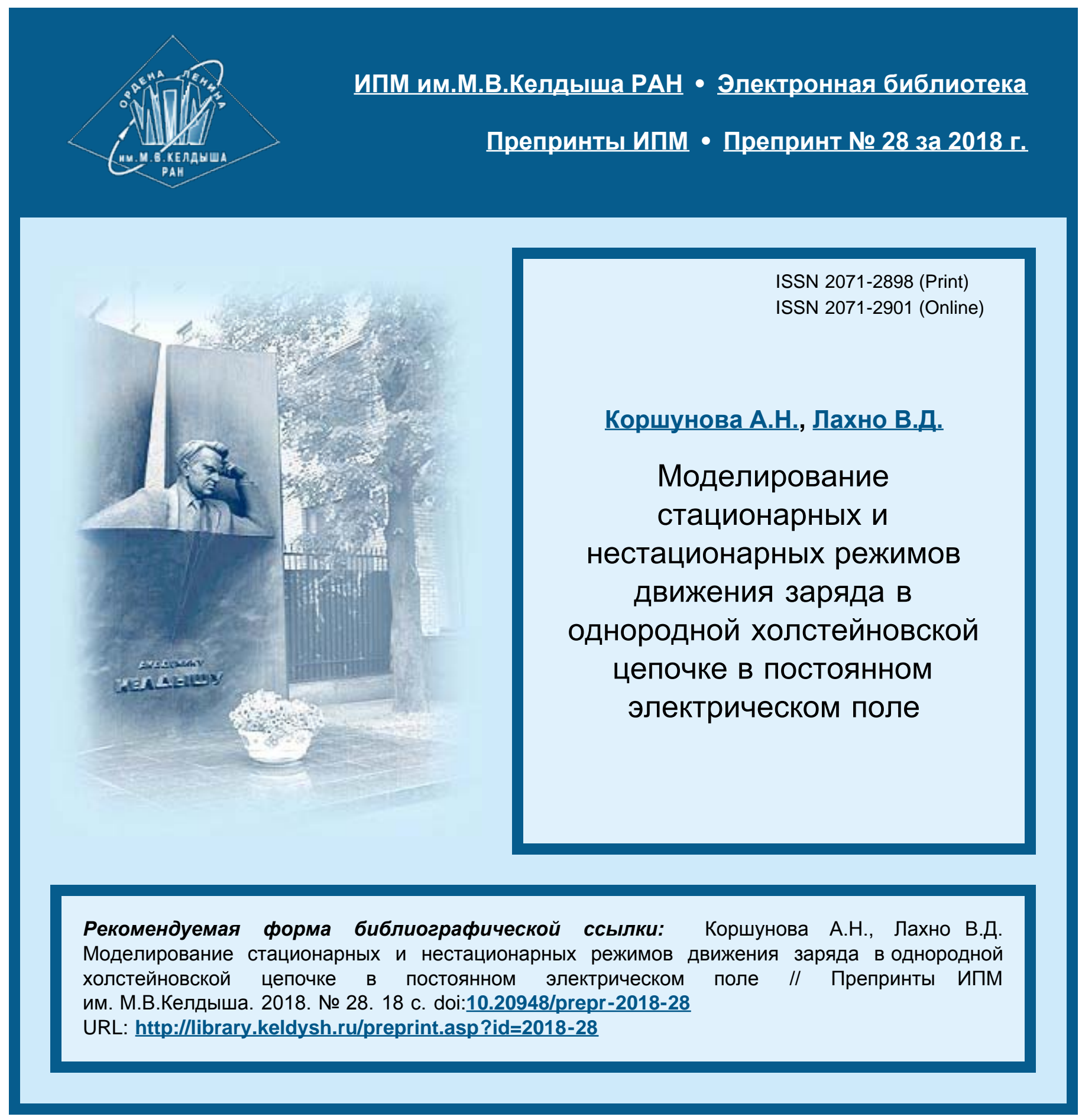




\author{
О р д е н а Л е н и н \\ ИНСТИТУТ ПРИКЛАДНОЙ МАТЕМАТИКИ \\ имени М.В.Келдыша \\ Российской академи инаук
}

А.Н. Коршунова, В.Д. Лахно

\title{
Моделирование стационарных и нестационарных режимов движения заряда в однородной холстейновской цепочке в постоянном электрическом поле
}




\section{А.Н. Кориунова, В.Д. Лахно}

Моделирование стационарных и нестационарных режимов движения заряда в однородной холстейновской цепочке в постоянном электрическом поле.

Проведено численное моделирование движения заряда в холстейновской молекулярной цепочке в постоянном электрическом поле. Показано, что при выбранных параметрах цепочки существует возможность равномерного движения заряда в постоянном электрическом поле на очень большие расстояния, на сотни тысяч сайтов. Движение заряда с постоянной скоростью возможно для небольших значений напряжённости электрического поля. С увеличением значения напряжённости электрического поля заряд переходит в колебательный режим движения с блоховскими осцилляциями. Показано хорошее соответствие теоретической и численной зависимости скорости движения заряда с постоянной скоростью от напряжённости электрического поля.

Ключевые слова: ДНК, нанобиоэлектроника, полярон, модель Холстейна, перенос заряда.

\section{A.N. Korshunova, V.D. Lakhno \\ Modeling of stationary and nonstationary regimes of charge transfer in a homogeneous Holstein chain in a constant electric field.}

Numerical simulation of charge transfer in a Holstein molecular chain in a constant electric field was carried out. It is shown that for selected chain parameters, there is the possibility of uniform charge transfer in a constant electric field over very long distances, to hundreds of thousands of sites. The movement of a charge with a constant velocity is possible for small values of the electric field intensity. At increasing the value of the electric field intensity, the moving of charge transforms into an oscillatory mode of motion with Bloch oscillations. A good agreement between the theoretical and numerical dependence of the uniform charge velocity on the electric field intensity is shown.

Key words: DNA, nanobioelectronics, polaron, Holstein model, charge transfer.

Работа выполнена с использованием вычислительных ресурсов Межведомственного суперкомпьютерного центра Российской академии наук (МСЦ РАН). Работа поддержана проектом: РФФИ №16-07-00305. 


\section{1. Введение}

Изучение движения зарядов в одномерных молекулярных цепочках является проблемой, актуальность которой связана с возможностью их использования в качестве проводов в наноэлектронных устройствах. Носителями тока в одномерных цепочках являются самозахваченные электронные состояния, которые имеют вид поляронных образований. Вопрос о возможности переноса энергии и заряда локализованными возбуждениями - солитонами и поляронами - в таких биологических молекулах, как белки, был поставлен Давыдовым [1] - [4]. В связи с развитием молекулярной нанобиоэлектроники, основной задачей которой является конструирование электронных устройств на основе биологических молекул [5] - [6], всё больший интерес вызывают проблемы транспорта заряда в таких протяжённых молекулах, как ДНК [7] - [15].

В настоящей работе приводятся результаты численного моделирования движения заряда в полинуклеотидной холстейновской молекулярной цепочке в постоянном электрическом поле. Ранее, в работе [16], этот вопрос был рассмотрен одним из авторов в случае, когда цепочка считалась непрерывной. Очевидно, полученные в [16] результаты могут быть непригодны в случае дискретной цепочки. Более того, в дискретном случае могут реализовываться такие режимы движения, которые отсутствуют в непрерывном случае.

\section{2. Динамическая модель дискретной холстейновской цепочки}

В используемой ниже модели полинуклеотидная цепочка рассматривается состоящей из $N$ сайтов. Каждый сайт представляет нуклеотидную пару, которая рассматривается как гармонический осциллятор [17]. Для моделирования квантовой динамики частицы в цепочке из $N$ нуклеотидных пар будем исходить из гамильтониана Холстейна, впервые рассмотревшего цепочку, каждый сайт которой представляет двухатомную молекулу [17], [18]:

$$
\begin{aligned}
H=- & \sum_{n}^{N} \nu(|n\rangle\langle n-1|+| n\rangle\langle n+1|)+\sum_{n}^{N} \alpha q_{n}|n\rangle\langle n|+ \\
& +\sum_{n}^{N} M \dot{q}_{n}^{2} / 2+\sum_{n}^{N} k q_{n}^{2} / 2+\sum_{n}^{N} e \mathcal{E} n|n\rangle\langle n|,
\end{aligned}
$$

где $\nu$ - матричный элемент перехода заряда между соседними сайтами (нуклеотидными парами), $\alpha$ - константа взаимодействия заряда со смещениями $q_{n}, M$ - эффективная масса сайта, $k$ - упругая постоянная.

Уравнения движения для гамильтониана $\hat{H}$ приводят к следующей си- 
стеме дифференциальных уравнений:

$$
\begin{aligned}
i \hbar \dot{b}_{n} & =-\nu\left(b_{n-1}+b_{n+1}\right)+\alpha q_{n} b_{n}+e \mathcal{E} a n b_{n} \\
M \ddot{q}_{n} & =-\gamma \dot{q}_{n}-k q_{n}-\alpha\left|b_{n}\right|^{2}
\end{aligned}
$$

где $b_{n}$ - амплитуда вероятности нахождения заряда на $n$-м сайте, $\sum_{n}\left|b_{n}\right|^{2}=1$. В классические уравнения движения (3) введена диссипация, определяемая коэффициентом трения $\gamma$.

Уравнения (2) являются уравнениями Шрёдингера для амплитуд вероятности $b_{n}$, описывающими эволюцию частицы в деформируемой цепочке, где $\hbar=h / 2 \pi, h$ - постоянная Планка, а уравнения (3) представляют классические уравнения движения, описывающие динамику нуклеотидных пар с учетом диссипации.

Перейдем в уравнениях (2), (3) к безразмерным переменным с помощью соотношений:

$$
\begin{aligned}
\eta & =\tau \nu / \hbar, \quad \omega^{2}=\tau^{2} K / M, \\
\omega^{\prime} & =\tau \gamma / M, \quad q_{n}=\beta u_{n}, E=\mathcal{E} e a \tau / \hbar, \\
\varkappa \omega^{2} & =\tau^{3}(\alpha)^{2} / M \hbar, \beta=\tau^{2} \alpha / M, \quad t=\tau \tilde{t},
\end{aligned}
$$

где $\tau$ - произвольный масштаб времени, связывающий время $t$ и безразмерную переменную $\tilde{t}$.

В безразмерных переменных (4) уравнения (2), (3) примут вид:

$$
\begin{aligned}
& i \frac{d b_{n}}{d \tilde{t}}=-\eta\left(b_{n+1}+b_{n-1}\right)+\varkappa \omega^{2} u_{n} b_{n}+E n b_{n}, \\
& \frac{d^{2} u_{n}}{d \tilde{t}^{2}}=-\omega_{n}^{\prime} \frac{d u_{n}}{d \tilde{t}}-\omega_{n}^{2} u_{n}-\left|b_{n}\right|^{2} .
\end{aligned}
$$

Введенная таким образом модель является простейшей моделью, описывающей динамику заряженной частицы в полинуклеотидной цепочке, в явном виде учитывающей диссипацию в рассматриваемой системе.

\section{3. Равномерное движения заряда}

Очевидно, что моделирование динамики частицы даже в однородной $(G)_{n}$ цепочке является задачей многопараметрической, что сразу же приводит к необходимости большого количества расчётов для различных значений параметров цепочки. Подбор параметров системы для моделирования конкретного режима поведения заряда в электрическом поле осуществляется как в соответствии с полученными результатами аналитического исследования системы в континуальном пределе [19], так и в результате проведённых численных исследований. Выбирая модельные па- 
раметры цепочек, мы можем исследовать движение заряда, характер распределения заряда со значительно большей вычислительной скоростью, чем для реальных ДНК цепочек.

Для моделирования движения заряда в электрическом поле были выбраны следующие значения безразмерных параметров: $\varkappa=1, \eta=1.276$, $\omega=1$. Для выбранных значений параметров варьируем значения трения в цепочке $\omega^{\prime}$ и значения напряжённости электрического поля $E$. Вычисления выполнялись стандартным методом Рунге-Кутта четвертого порядка. Для моделирования равномерного движения заряда в электрическом поле мы поместили в цепочку в начальный момент времени заряд, соответствующий стационарному решению уравнений (2), (3) в отсутствие внешнего поля, а именно: начальные значения $\left|b_{n}(0)\right|$ были выбраны в виде обратного гиперболического косинуса:

$$
\begin{aligned}
\left|b_{n}(0)\right| & =\frac{\sqrt{2}}{4} \sqrt{\frac{\varkappa}{|\eta|}} \operatorname{ch}^{-1}\left(\frac{\varkappa\left(n-n_{0}\right)}{4|\eta|}\right), \\
u_{n}^{0} & =\left|b_{n}(0)\right|^{2} / \omega_{n}^{2}, d u_{n}^{0} / d \tilde{t}=0 .
\end{aligned}
$$

Определим характерный размер распределения заряда в цепочке как:

$$
d(\tilde{t})=\sum\left|b_{n}(\tilde{t})\right|^{2} / \sum\left|b_{n}(\tilde{t})\right|^{4}=1 / \sum\left|b_{n}(\tilde{t})\right|^{4} .
$$

При выбранных значениях параметров цепочки характерный размер полярона в цепочке определяется выражением: $\lim _{\tilde{t} \rightarrow \infty} d(\tilde{t}) \approx 15$. То есть при таких параметрах цепочки полярон широкий, располагается на достаточно большом количестве сайтов. Значение $n_{0}$ (центр начального распределения заряда) в (6) выбиралось так, чтобы в начале вычислений полярон был достаточно далеко от концов цепочки. Аналогично и длина цепочки подбирается так, чтобы и в конце вычислений полярон не подошел слишком близко к концу цепочки. Поле включается "мгновенно"в начальный момент времени.

Вполне закономерно возникает вопрос, при каких значениях напряжённости электрического поля $E$ существует возможность равномерного движения заряда в цепочке для выбранных значений параметров. В работе [19] подробно рассмотрен вопрос о соотношении между равновесной скоростью полярона $V=v a / \tau$ и напряженностью электрического поля в континуальном пределе. Для недемпфированного движения нуклеотидных пар, когда трение не слишком велико $\omega>\omega^{\prime} / 2$, в [19] получено следующее соотношение между равновесной скоростью полярона $V$ и напря- 
женностью электрического поля $E$ :

$$
\begin{aligned}
E & =\varkappa \omega^{2} \omega^{\prime} V I, \quad E=\mathcal{E} \frac{e a \tau}{\hbar}, \\
I & =\frac{2 \eta}{\varkappa V^{4}} \int_{0}^{\infty} \frac{x^{4} / \operatorname{sh}^{2} x}{\left(x^{2}+c_{1}\right)^{2}+c_{2}^{2}} d x, \\
c_{1} & =\left(\frac{2 \pi \eta}{\varkappa V}\right)^{2}\left(\frac{\omega^{\prime 2}}{2}-\omega^{2}\right), \\
c_{2}^{2} & =\left(\frac{2 \pi \eta}{\varkappa V}\right)^{4} \omega^{\prime 2}\left(\omega^{2}-\left(\frac{\omega^{\prime}}{2}\right)^{2}\right) .
\end{aligned}
$$

В случае демпфированного движения нуклеотидных пар (для $\omega^{\prime} / 2>$ $\omega)$ величина $I$, согласно [19], определяется соотношением:

$$
\begin{aligned}
& I=\frac{2 \eta}{\varkappa V^{4}} \int_{0}^{\infty} \frac{x^{4} / \operatorname{sh}^{2} x}{\left(x^{2}+c_{1}^{2}\right)\left(x^{2}+c_{2}^{2}\right)} d x, \\
& c_{1}=\frac{2 \pi \eta}{\varkappa V}\left(\frac{\omega^{\prime}}{2}-\sqrt{\left(\frac{\omega^{\prime}}{2}\right)^{2}-\omega^{2}}\right), \\
& c_{2}=\frac{2 \pi \eta}{\varkappa V}\left(\frac{\omega^{\prime}}{2}+\sqrt{\left(\frac{\omega^{\prime}}{2}\right)^{2}-\omega^{2}}\right) .
\end{aligned}
$$

Предельный случай, когда трение отсутствует, был рассмотрен в работе [20]. В данной работе мы рассмотрим зависимость скорости полярона $V$ от напряжённости электрического поля $E$ для различных значений трения $\omega^{\prime}$.

На Рис. 1 приведены зависимости скорости солитона от напряженности электрического поля $E$ для различных значений параметра $\omega^{\prime}$ при $\varkappa=1$, определяемые (8), (9).

Четыре левые кривые, представленные на Рис.1, соответствуют демпфированному движению нуклеотидных пар (9): $\omega^{\prime} / 2>\omega$, а именно $\omega^{\prime}=$ $6,4,3,2$. Следующие пять кривых соответствуют недемпфированному движению нуклеотидных пар (8): $\omega^{\prime} / 2<\omega, \omega^{\prime}=1.5,1,0.5,0.1,0.01$. Рассмотренный в [20] предельный случай $\omega^{\prime}=0$ соответствует крайней правой кривой на Рис. 1 , а предельному случаю $\omega^{\prime}=\infty$ соответствует ось ординат. Согласно Рис. 1 равномерное движение солитона возможно только в интервале $0<E<E_{\max }\left(\omega^{\prime}\right)$. На каждой кривой ветвь, для которой $d V / d E=V_{E}^{\prime}>0$, соответствует устойчивому движению солитона, а ветвь с $V_{E}^{\prime}<0$ - неустойчивому движению. Из (8), (9) следует, что наличие в 


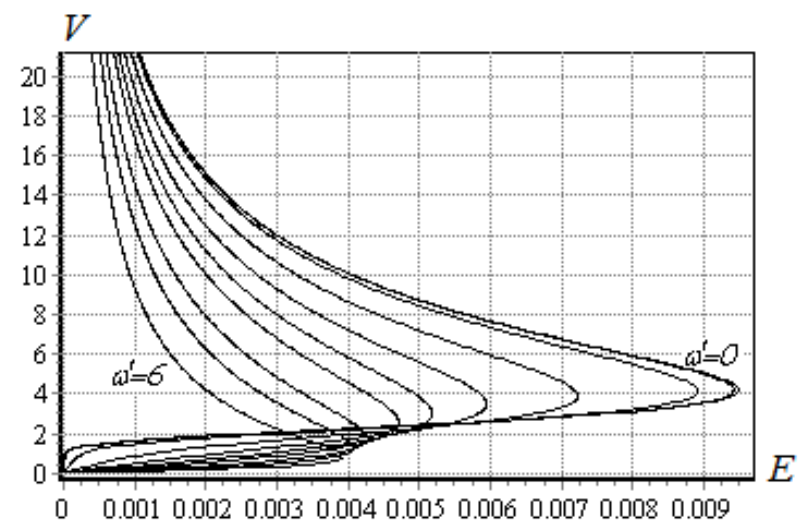

Рuс. 1. Зависимость скорости полярона $V$ от напряжённости электрического поля $E$ при $\varkappa=1$ для различных значений параметра $\omega^{\prime}$ $\left(\omega^{\prime}=6,4,3,2,1.5,1,0.5,0.1,0.01,0.\right) ;$
$\eta=1.276, \omega=1$.

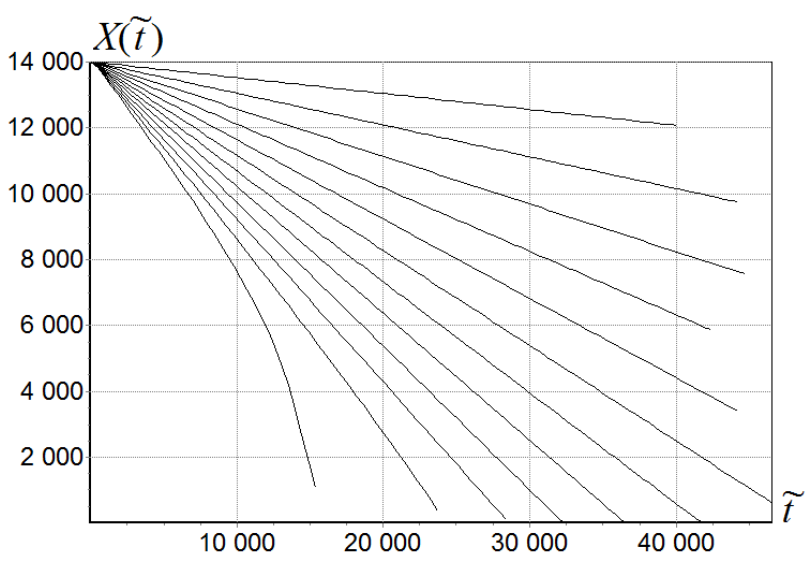

Puc. 2. Графики функции $X(\widetilde{t})$ при $\varkappa=1, \eta=1.276, \omega=1, \omega^{\prime}=1$ и

значениях напряжённости электрического поля

$E=0.0001, E=0.0002, E=$ $0.0003, \ldots E=0.0012$ для $\widetilde{t}=47000$. Длина цепочки $N=15001$ сайтов. Верхний график соответствует значению $E=0.0001$.

системе трения приводит к появлению омического участка, т.е. линейной зависимости $V(E)$ при малых $E$ на устойчивых ветвях кривых $V(E)$.

На Рис. 2 показаны графики функции

$$
X(\widetilde{t})=\sum_{n}\left|b_{n}(\widetilde{t})\right|^{2} \cdot n,
$$

описывающие движение центра масс частицы. Представленные графики демонстрируют линейную зависимость от $\widetilde{t}$ почти для всех значений напряжённости электрического поля $E$ (см. Рис. 2). Хорошо видно, что самый левый график функции $X(\widetilde{t})$, для $E=0.0012\left(\mathcal{E}=2.256 \cdot 10^{3} \mathrm{~B} / \mathrm{cm}\right)$, на Рис. 2 заметно отклоняется от прямой линии, это означает, что заряд переходит в колебательный режим движения. Таким образом, показано, что для значений поля $E>0.0011$ при выбранных параметрах цепочки равномерного движения заряда не существует.

На Рис. 3 показаны графики функций $\left|b_{n}(\widetilde{t})\right|$ и $X(\widetilde{t})$ для напряженности электрического поля $E=0.001$. В начальный момент времени полярон находится на сайте с номером $n 0=601$. Это пример равномерного движения полярона по цепочке. Также представленному на Рис. 3 примеру соответствует третий слева график на Рис. 2, тоже соответствующий значению $E=0.001$.

На Рис. 4 и Рис. 5 изображены участки зависимости скорости солито- 


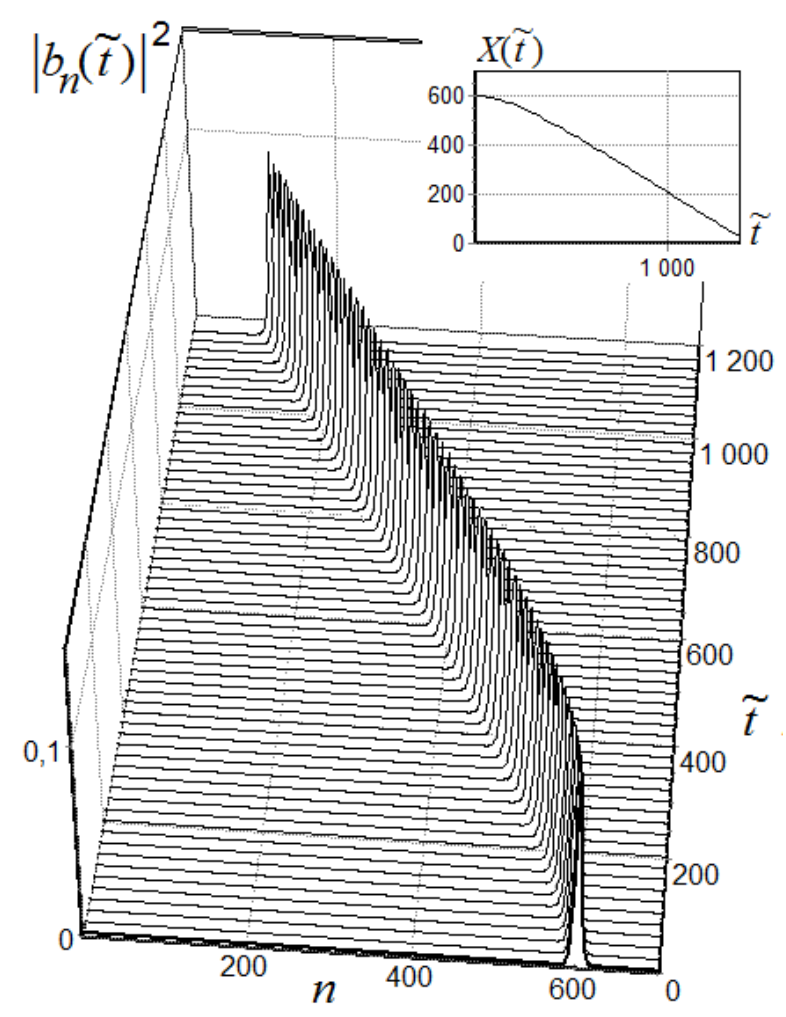

Puc. 3. Графики функций $\left|b_{n}(\widetilde{t})\right|^{2}$ и $X(\widetilde{t})$ при $\varkappa=1, \eta=1.276, \omega=1, \omega^{\prime}=1$ и значении напряжённости электрического поля $E=0.001$, для (безразмерного) времени $\widetilde{t}=1200$. Длина цепочки $N=701$ сайтов.

на $V$ от внешнего электрического поля $E$, которые дают детальное представление устойчивого движения солитона $\left(d V / d E=V_{E}^{\prime}>0\right)$. Рис. 5 представляет собой увеличенную часть Рис. 4. Из приведенных графиков видно, что область линейной зависимости расширяется с ростом величины $\omega^{\prime}$. При $\omega^{\prime} \gtrsim 1$ (для $\varkappa=1$ ) область линейной зависимости скорости солитона от поля простирается вплоть до величины критического значения электрического поля, при котором равномерное движение солитона становится невозможным. В этом случае вплоть до очень больших напряжённостей электрического поля закон Ома выполняется с высокой точностью.

На Рис. 4 представлено сравнение численной $(\bullet)$ и "теоретической" скорости солитона $V$ от напряжённости электрического поля $E$. Заметим, что на этом рисунке предельному случаю $\omega^{\prime}=0$ соответствует крайняя левая кривая.

Согласно теоретическим выводам, следующим из Рис. 1 и Рис. 4, равномерное движение заряда возможно только в интервале $0<E<E_{\max }\left(\omega^{\prime}\right)$. На представленных графиках точка $E_{\max }\left(\omega^{\prime}\right)$ соответствует точке перегиба кривой $V(E)$. Численные же эксперименты показывают, что этот интервал $0<E<E_{\text {max-numerical }}\left(\omega^{\prime}\right)$ значительно меньше, чем $0<E<E_{\max }\left(\omega^{\prime}\right)$, и для $E>E_{\text {max-numerical }}\left(\omega^{\prime}\right)$ равномерного движения заряда уже не наблюдается, 


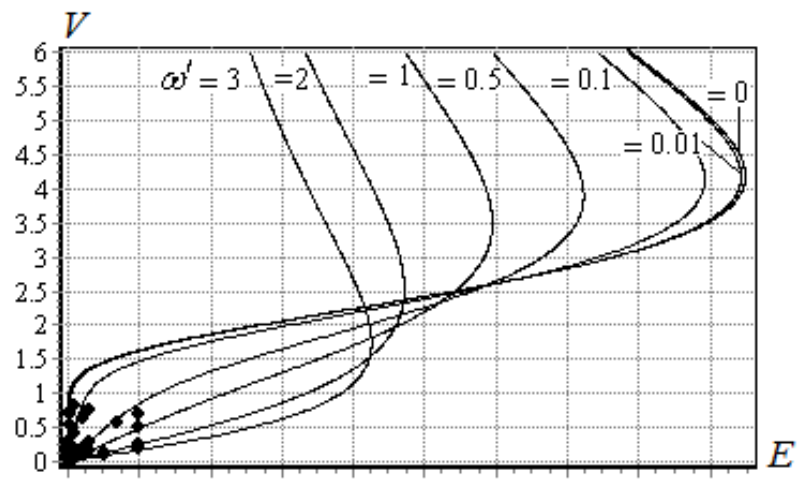

$\begin{array}{lllllllllll}0 & 0.001 & 0.002 & 0.003 & 0.004 & 0.005 & 0.006 & 0.007 & 0.008 & 0.009\end{array}$

Puc. 4. Графики зависимости

скорости солитона $V$ от напряжённости электрического поля $E$ при $\varkappa=1$, $\omega^{\prime}=3,2,1,0.5,0.1,0.01,0$. $\eta=1.276, \omega=1$, соответствующие устойчивому движению солитона (при $d V / d E=V_{E}^{\prime}>0$ ).

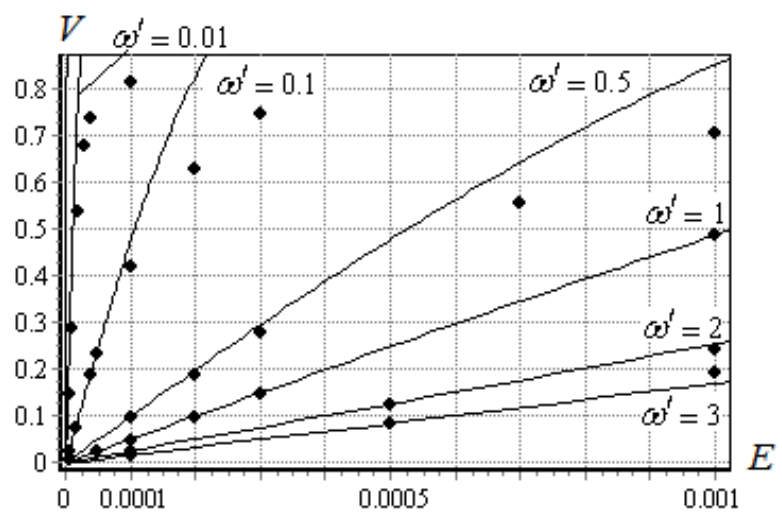

Pис. 5. Сравнение "теоретической"(-) (8), (9) и численной $(\bullet)$ скорости солитона $V$ от напряжённости электрического поля $E$ при $\varkappa=1$, $\omega^{\prime}=3,2,1,0.5,0.1,0.01,0$. $\eta=1.276, \omega=1$ для участков линейной зависимости $V(E)$.

см. Рис. 2.

\section{4. Неравномерное движения заряда}

Вычисления показывают, что для $E>E_{\text {max-numerical }}\left(\omega^{\prime}\right)$ (так же и для $\left.E>E_{\max }\left(\omega^{\prime}\right)\right)$ в начале движения заряд испытывает блоховские осцилляции как целое, затем заряд теряет свою первоначальную форму. Чем больше значение $E$ и чем больше оно превосходит $E_{\text {max-numerical }}\left(\omega^{\prime}\right)$, тем быстрее начальное распределение (в виде обратного гиперболичекого косинуса вида (6)) теряет свою первоначальную форму. Далее заряд движется по направлению поля, совершая колебания, период которых близок к периоду блоховских осцилляций $T_{B L}=2 \pi / E$. Никаких особенностей в характере движения частицы в поле при "переходе" $E$ через $E_{\max }\left(\omega^{\prime}\right)$ не происходит.

Как и в описанном выше случае моделирования равномерного движения заряда в электрическом поле, для моделирования неравномерного движения мы помещали в цепочку в начальный момент времени заряд, соответствующий стационарному решению уравнений (2), (3) в отсутствие внешнего поля. Начальные значения $\left|b_{n}(0)\right|$ тоже были выбраны в виде обратного гиперболического косинуса вида (6). Но в данном случае были выбраны бо́льшие значения напряжённости электрического поля $E$.

На Рис. 6 представлены графики функции $X(\widetilde{t})$ для значений напряжённости электрического поля $E$ начиная со значения $E=0.001$. Это- 
му значению $E=0.001$ на Рис. 6 соответствует единственный график в виде прямой линии, что указывает на равномерное движение заряда по цепочке. Все остальные графики на Рис. 6 указывают на колебательный режим движения заряда по цепочке. Период блоховских осцилляций для $E=0.001$ равен $T_{B L}=2 \pi / E \approx 6283$. Из чего следует, что график для $E=0.001$ показан за время, соответствующее примерно 3.6 блоховским периодам. То есть мы видим, что этот график действительно указывает на равномерное движение, а не является частью графика при колебательном движении полярона. Заметим, что на Рис. 2 тоже есть график, соответствующий $E=0.001$, это третий график слева, и он показан за время $\widetilde{t} \approx 28000$, что равно примерно 4.5 блоховским периодам.

На графиках рисунка 6, соответствующих колебательному режиму движения, хорошо видно, что с увеличением значения напряжённости электрического поля период колебаний уменьшается и всегда примерно равен $T_{B L}=2 \pi / E$. Обратим внимание на график функции $X(\widetilde{t})$ для $E=0.002$ на Рис. 6 , это самый нижний график, не считая графика для $E=0.001$. Его вид для $\widetilde{t}>10000$ некорректен в том смысле, что заряд уже начал отражаться от левого края цепочки, и это хорошо видно на графике. Кроме того, при колебательном режиме движения, если в начальный момент времени в движении участвует заряд в целом, как в данном случае, то центр масс заряда смещается на максимальную блоховскую амплитуду $A_{B L}=4 \eta / E$. Для $E=0.002$ максимальная блоховская амплитуда $A_{B L}=4 \eta / E \approx 2500$, а на графике мы видим, что центр массы полярона сместился сразу на 4000 сайтов. На самом деле для этого значения напряжённости электрического поля $E=0.002$ начальный полярон при мгновенном включении электрического поля двигается почти равномерно, слегка оседая по высоте, и только пройдя примерно 1500 целиком входит в блоховские осцилляции. Только начиная со значения электрического поля $E=0.005$ полярон после начала движения сразу уходит на максимальную блоховскую амплитуду $A_{B L}=4 \eta / E$.

На Рис. 7 показан пример движения полярона в короткой цепочке длиной $N=701$ для большого значения напряжённости электрического поля $E=0.03$. Период блоховских осцилляций равен $T_{B L}=2 \pi / E \approx 209$ для $E=0.03$, максимальная блоховская амплитуда $A_{B L}=4 \eta / E \approx 170$. Начальный полярон вида (6) имеет характерный размер примерно 15 и высоту около 0.1. При отсутствии электрического поля такое начальное поляронное состояние практически сохраняет свою форму и не сдвигается из своего начального положения как угодно долго, так как выбрана цепочка с большим трением. Заметим, что при отсутствии трения или при очень маленьком трении в цепочке полярон сохранит своё начальное положение только в случае, когда он находится в центре цепочки, см. [21], [22]. Поскольку выбрана цепочка с большим трением и начальный полярон не 


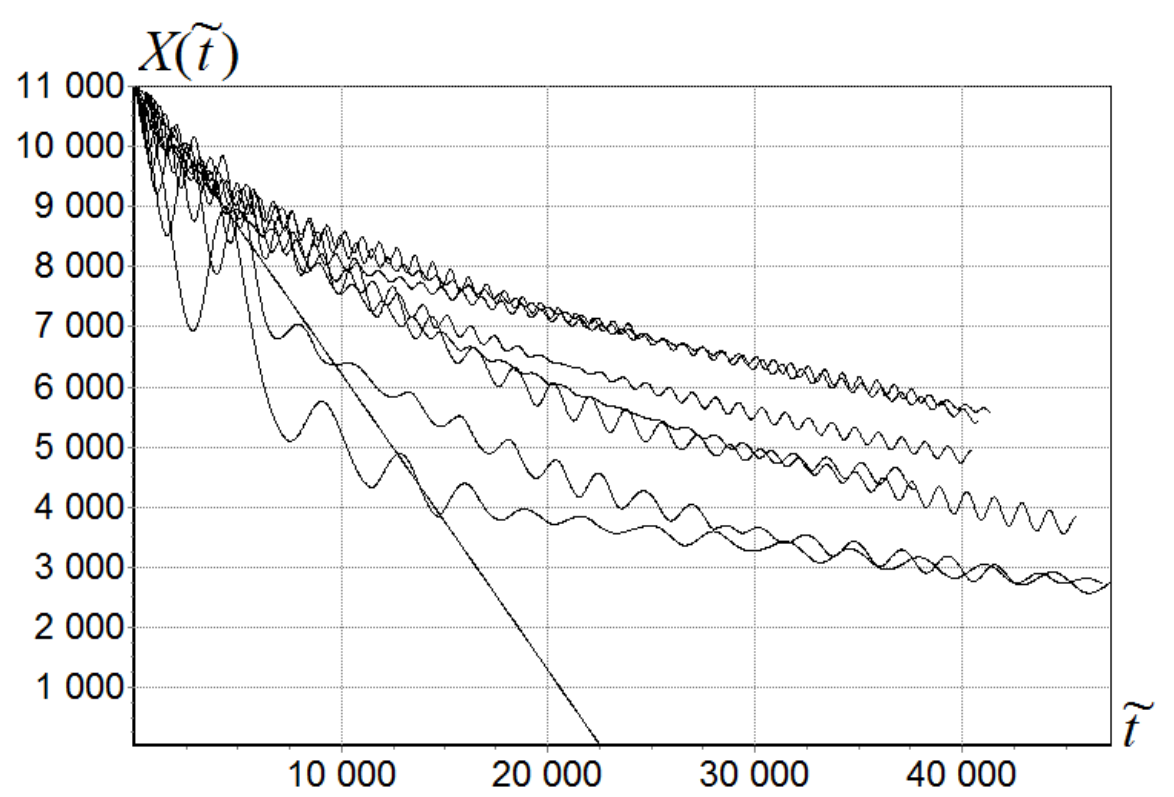

Puc. 6. Графики функции $X(\widetilde{t})$ при $\varkappa=1, \eta=1.276, \omega=1, \omega^{\prime}=1$ и значениях напряжённости электрического поля $E=0.001, E=0.002, E=0.003, \ldots E=0.008$ для (безразмерного) времени $\widetilde{t}=47000$. Длина цепочки $N=12001$ сайтов.

сдвигается из начального положения при отсутствии поля, то при мгновенном включении поля в процессе первой осцилляции заряд локализуется в пределах примерно одной максимальной блоховской амплитуды по направлению поля.

Графики функций $\left|b_{n}(\widetilde{t})\right|^{2}$ наглядно демонстрируют, как начальный полярон вида (6) быстро разваливается, и, совершая блоховские осцилляции, движется по направлению поля в цепочке. Также на Рис. 7 хорошо заметно, как в ходе последней по времени пятой осцилляции заряд отразился от конца цепочки, а не от границы, обусловленной полем, как в предыдущих осцилляциях.

Характер движения заряда в электрическом поле сильно зависит от начального распределения заряда по цепочке. На Рис. 7 представлен пример движения заряда по цепочке из начального поляронного состояния вида (6). Посмотрим, как ведёт себя в поле заряд, находящийся на одном сайте. Поместим заряд на один сайт в центре цепочки. На рисунке 8 показано распределение такого разряда по цепочке в отсутствие электрического поля. Прямая линия в центре графика показывает заряд величиной в единицу в центре цепочки при левой шкале 0.12. Хорошо видно, как заряд быстро растекается по всей цепочке, отражается от концов цепочки, и, постепенно распределяется равномерно по цепочке.

Совершенно по-другому распределяется по цепочке единичный заряд в электрическом поле. На Рис. 9 показан следующий пример. В начальный момент времени заряд находится на одном сайте в центре цепочки, 


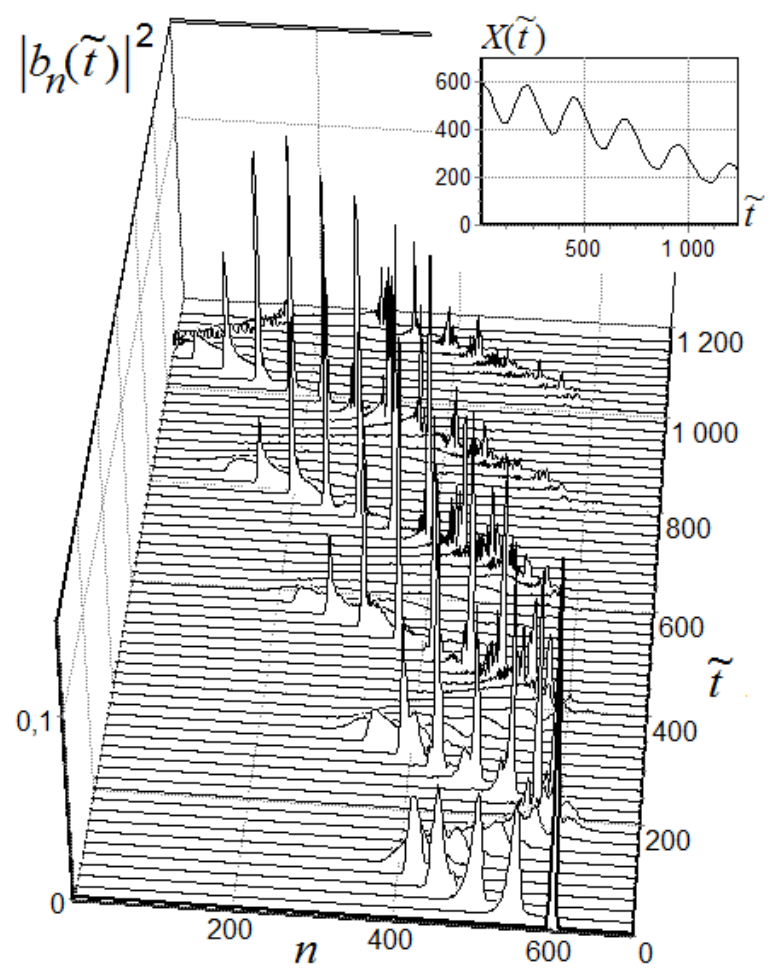

Puc. 7. Графики функций $\left|b_{n}(\widetilde{t})\right|^{2}$ и $X(\widetilde{t})$ при $\varkappa=1, \eta=1.276, \omega=1, \omega^{\prime}=1$, значение напряжённости электрического поля $E=0.03$, для (безразмерного) времени $\widetilde{t}=1200$. Длина цепочки $N=701$ сайтов.

поле включается мгновенно в начальный момент времени, значение напряжённости электрического поля $E=0.03$, такое же, как в примере на Рис. 7. Графики функции $\left|b_{n}(\widetilde{t})\right|^{2}$ показывают, что заряд не растекается по всей цепочке, как при отсутствии поля.

Начальный единичный заряд сначала растекается в обе стороны от центра цепочки, но локализуется электрическим полем в пределах примерно по одной максимальной блоховской амплитуде в обе стороны от центра цепочки. Далее заряд движется, осциллируя по цепочке, с периодом колебаний, близким к блоховскому периоду для заданного значения напряжённости электрического поля $E=0.03$. Характер и скорость движения в этом случае значительно отличаются от показанных в примере на Рис. 7.

Численное моделирование показывает, что характер движения и скорость заряда в поле сильно зависят от характерного размера как начального распределения заряда в цепочке, так и от характерного размера устоявшегося полярона в цепочке при заданных параметрах. В примерах на рисунках 7 и 9 показаны отличия в распределении заряда по цепочке при колебательном режиме движения в зависимости от начального распределения.

Для $\varkappa>1$ (при $\eta=1.276)$ существует возможность при одинаковых 


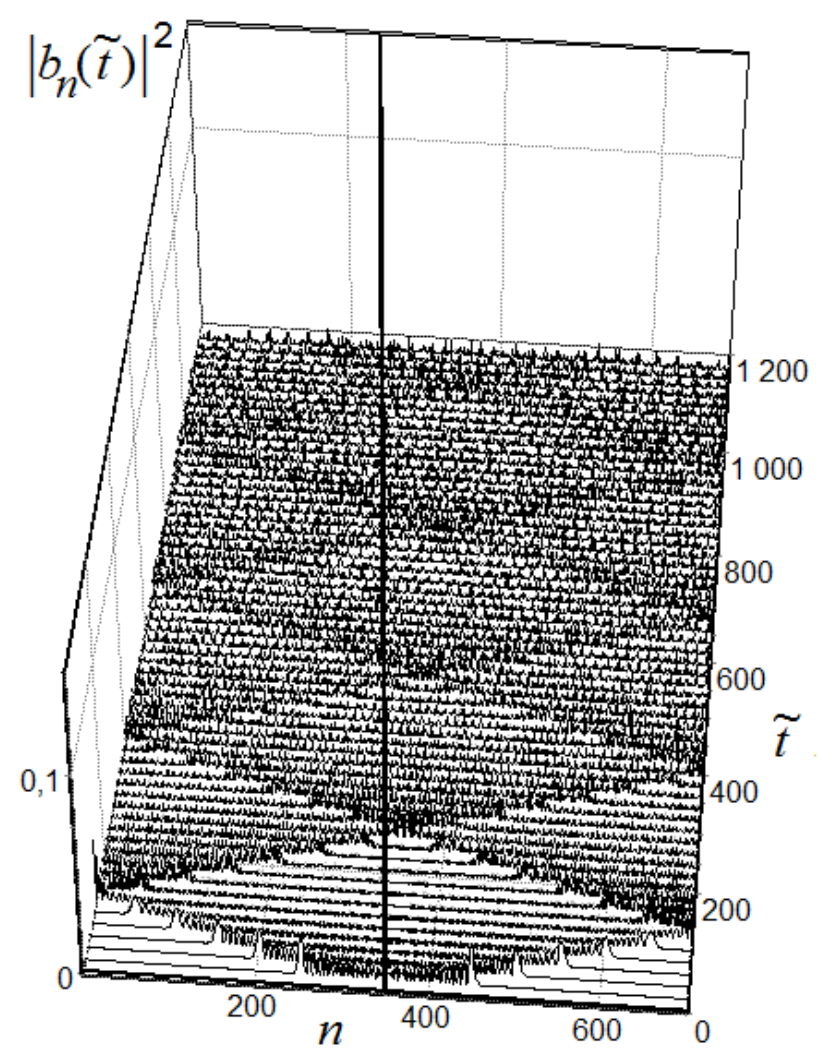

Puс. 8. Графики функций $\left|b_{n}(\widetilde{t})\right|^{2}$ и $X(\widetilde{t})$ при $\varkappa=1, \eta=1.276, \omega=1$, $\omega^{\prime}=1$ при отсутствии

электрического поля, для (безразмерного) времени $\widetilde{t}=1200$. Длина цепочки $N=701$ сайтов. В начальный момент времени заряд

находится на одном сайте в центре цепочки, $u_{n}^{0}=0$ для всех $n$.

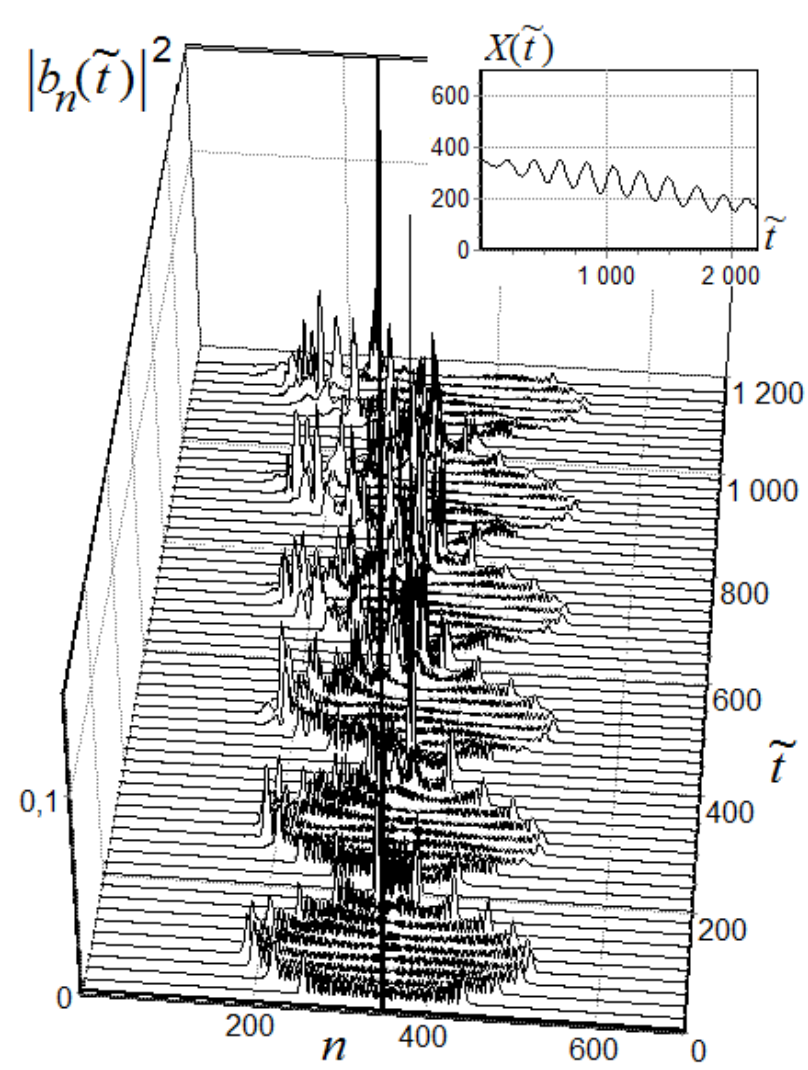

Puc. 9. Графики функции $\left|b_{n}(\widetilde{t})\right|^{2}$ и $X(\widetilde{t})$ при $\varkappa=1, \eta=1.276, \omega=1$, $\omega^{\prime}=1$, значение напряжённости электрического поля $E=0.03$ для (безразмерного) времени $\widetilde{t}=1200$. Длина цепочки $N=701$ сайтов. В начальный момент времени заряд находится на одном сайте в центре цепочки, $u_{n}^{0}=0$ для всех $n$.

параметрах цепочки и одном и том же напряжении электрического поля получить и равномерное движение заряда и колебательное в зависимости от начального распределения заряда, например изменяя начальное распределение вида (6). Возьмем начальные значения $\left|b_{n}(0)\right|$ виде растянутого обратного гиперболического косинуса:

$$
\left|b_{n}(0)\right|=\frac{\sqrt{2}}{4} \sqrt{\frac{\varkappa}{\xi|\eta|}} \operatorname{ch}^{-1}\left(\frac{\varkappa\left(n-n_{0}\right)}{4 \xi|\eta|}\right),
$$

$\xi$ - коэффициент растяжения.

Возьмем такое значение напряженности электрического поля $E$, при котором существует равномерное движение заряда при начальных значениях $\left|b_{n}(0)\right|$ в виде нерастянутого обратного гиперболического косину- 
са вида (6). При выбранном значении $E$ будем постепенно увеличивать или уменьшать значение $\xi$. В зависимости от выбранных параметров до некоторого значения $\xi(\xi \neq 1)$ начальный растянутый обратный гиперболический косинус вида (11), несмотря на воздействие поля, достаточно быстро принимает форму нерастянутого обратного гиперболического косинуса вида (6) (или устоявшегося распределения заряда). В этом случае заряд после небольших колебаний в начальное время далее движется по цепочке равномерно.

Если значение $\xi$ взято достаточно отличающимся от единицы (для выбранного значения $E$ ), то заряд теряет свою первоначальную форму и, осциллируя, движется по цепочке по направлению поля. В этом случае движение заряда может быть аналогичным движению заряда для случая, описанного выше, а именно, когда начальное распределение взято в виде нерастянутого обратного гиперболического косинуса вида (6), но величина напряженности электрического поля $E$ превышает значения, при которых существует равномерное движение заряда. Мы написали “может быть аналогичным”, так как характер распределения заряда может заметно изменяться в зависимости от параметров цепочки.

\section{5. Выводы}

Мы рассмотрели моделирование равномерного движения заряда для следующих значений параметров цепочки: $\varkappa=1, \eta=1.276$. При выбранных значениях параметров существует возможность движения заряда с постоянной скоростью по направлению поля в цепочке. В процессе движения заряд сохраняет свою форму. Такое равномерное движение и достаточно хорошее (Рис. 4) соответствие "теоретической" и численной зависимости скорости заряда $V$ от напряженности внешнего электрического поля $E$ обусловлено тем, что при таких параметрах цепочки характерный размер полярона в цепочке $\lim _{\tilde{t} \rightarrow \infty} d(\tilde{t}) \approx 15$, это широкий полярон, располагается на достаточно большом количестве сайтов.

При увеличении $\varkappa>1$ (для $\eta=1.276$ ) устоявшееся поляронное распределение заряда становится "у́же". Вследствие этого при $\varkappa=2$ и более становятся хорошо заметны колебания Пайерлса-Набарро, обусловленные дискретностью решетки [20]. Таким образом, при $\varkappa \geq 2$ равномерное движение заряда носит слабо колебательный характер, при котором форма заряда сохраняется "в периоде".

Вычисления показывают, что равномерное движение заряда при $\varkappa=2$ и $\varkappa=3$ существует, так же как и для $\varkappa=1$. А именно, равномерное движение заряда возможно только в интервале $E<E_{\text {max-numerical }}\left(\omega^{\prime}\right)$. Для $E>E_{\text {max-numerical }}\left(\omega^{\prime}\right)$ равномерного движения заряда не наблюдается, начальная форма распределения заряда "разваливается", заряд, совершая 
колебательные движения с блоховским периодом, движется по цепочке по направлению поля, причем в начале движения амплитуда колебаний заряда близка к максимальной блоховской амплитуде колебаний.

Однородной последовательности из PolyG/PolyC нуклеотидных пар соответствуют следующие значения безразмерных параметров: константа связи $\varkappa=4$, матричные элементы перехода частицы с одного сайта на другой $-\eta=1.276$, см. [23], [24]. Таким параметрам соответствует устоявшееся поляронное распределение заряда (см. [25]), располагающееся всего на нескольких сайтах, то есть очень “узкий пик”. В связи с этим возникают сложности при моделировании равномерного движения заряда в такой цепочке. Например, для $\omega=1$ и $\omega^{\prime}=1$ находится одно значение $E=0.09$, при котором заряд движется с постоянной скоростью по направлению поля в цепочке, совершая небольшие колебания и сохраняя свою форму. При малых значениях напряженности электрического поля $E<0.09$ заряд совсем не движется (стоит на месте), а при увеличении $E>0.09$ заряд “разваливается" и, совершая колебательные движения, движется по цепочке по направлению поля. Как и в ранее рассмотренных случаях, эти колебания соответствуют блоховским колебаниям.

Ранее в работе [26] нами были рассмотрены примеры блоховских осцилляций в модели Холстейна в зависимости от различных значений параметра . Аналогичные результаты были получены позже в работе [27] при исследовании блоховских осцилляций в модели Пейрарда-БишопаХолстейна. Кроме описанных здесь вариантов движения и распределения заряда по цепочке, в работе [28] была показана возможность неравновесного движения заряда в PolyG/PolyC цепочке ДНК.

Из проведенных расчетов следует, что в рассматриваемой системе могут реализоваться сложные динамические режимы, зависящие от выбранных параметров системы: от частоты, от коэффициента трения, от длины цепочки, от характерного размера устоявшегося полярона в цепочке, который обусловлен безразмерными параметрами связи электрона и решётки в цепочке. При фиксированных параметрах системы, изменяя только начальное распределение заряда и величину напряжённости электрического поля, можно наблюдать самые разнообразные режимы движения и распределения заряда в цепочке.

\section{Библиографический список}

1. Davydov A.S. The theory of contraction of proteins under their excitation. // J. Theor. Biology. 1973. Vol. 38. Issue 3. P. 559-569. https://doi.org/10.1016/0022-5193(73)90256-7

2. Davydov A.S. Solitons and energy transfer along protein molecules.// J. 
Theor. Biology. 1977. Vol. 66. P. 379-387. https://doi.org/10.1016/00225193(77)90178-3

3. Davydov A.S. // Solitons in Molecular systems. Reidel Publ. Comp., Boston, USA, 1985. $413 \mathrm{p}$.

4. Scott A.C. Davydov's soliton. // Phys. Rep. 1992. Vol. 217. Issue 1. P. 1-67. https://doi.org/10.1016/0370-1573(92)90093-F

5. Lakhno V.D. DNA nanobioelectronics. // Int. J. Quantum Chem. 2008. Vol. 108. P. 1970-1981. https://doi.org/10.1002/qua.21717

6. Nanobioelectronics - for Electronics, Biology and Medicine. Eds. Offenhausser A., Rinald N.Y.: Springer, 2009.

7. Storm A.J., Van Noort J., De Vries S., Dekker C. Insulating behavior for DNA molecules between nanoelectrodes at the $100 \mathrm{~nm}$ length scale. // Appl. Phys. Lett. 2001. Vol. 79. P. 3881-3883. https://doi.org/10.1063/1.1421086

8. De Pablo P.J., et. al. Absence of dc-Conductivity in $\lambda$ DNA. // Phys. Rev. Lett. 2000. Vol. 85. P. 4992-4995. https://doi.org/10.1103/PhysRevLett.85.4992

9. Fink H.W., Schönenberger C. Electrical conduction through DNA molecules. // Nature. 1999. Vol. 398. P. 407-409. https://doi.org/10.1038/18860

10. Okahata Y., Kobayashi T., Tanaka K., Shimomura M. Anisotropic Electric Conductivity in an Aligned DNA Cast Film. // J. Am. Chem. Soc. 1998. Vol. 120. P. 6165-6166. https://doi.org/10.1021/ja980165w

11. Porath D., Bezryadin A., De Vries S., Dekker C. Direct measurement of electrical transport through DNA molecules. // Nature. 2000. Vol. 403. P. 635-638. https://doi.org/10.1038/35001029

12. Cai L., Tabata H., Kavai T. Self-assembled DNA networks and their electrical conductivity. // Appl. Phys. Lett. 2000. Vol. 77. P. 3105-3106. https://doi.org/10.1063/1.1323546

13. Yoo K.-H. et. al. Electrical Conduction through $\operatorname{Poly}(\mathrm{dA})-\operatorname{Poly}(\mathrm{dT})$ and Poly(dG)-Poly(dC) DNA Molecules. // Phys. Rev. Lett. 2001. Vol. 87. P. 198102. https://doi.org/10.1103/PhysRevLett.87.198102

14. Kasumov A.Y., et.al. Proximity-Induced Superconductivity in DNA. // Science. 2001. Vol. 291. Issue 5502. P. 280-282. https://doi.org/10.1126/science.291.5502.280 
15. Chepeliaskii et. al. Conduction of DNA molecules attached to a disconnected array of metallic Ga nanoparticles. // New J. Phys. 2011. Vol. 13. P. 063046. 10 p. https://doi.org/10.1088/1367-2630/13/6/063046

16. Lakhno V.D. Davydov's Solitons in DNA, Chapter in "Self-Organization of Molecular Systems - From Molecules and Clusters to Nanotubes and Proteins."Eds. N.Russo, V.Ya. Antonchenko, E. Kryachko, Springer, 2009. 400 p. ISBN: 978-90-481-2483-1, P. 255-273.

17. Lakhno V.D. Soliton-like solutions and electron transfer in DNA.// J.Biol. Phys. 2000. Vol. 26. P. 133-147. https://doi.org/10.1023/A:1005275211233

18. Holstein T. Studies of polaron motion: Part II. The "small"polaron. // Ann. Phys. 1959. Vol. 8. Issue 3. P. 343-389. https://doi.org/10.1016/00034916(59)90003-X

19. Lakhno V.D. Davydov's solitons in homogeneous nucleotide chain. // Int. J. Quant. Chem. 2010. Vol. 110. Issue 1. P. 127-137. https://doi.org/10.1002/qua.22264

20. Lakhno V.D., Korshunova A.N. Electron motion in a Holstein molecular chain in an electric field. // Eur. Phys. J. B. 2011. Vol. 79. P. 147-151. https://doi.org/10.1140/epjb/e2010-10565-2

21. Коршунова А.Н., Лахно В.Д. Особенности движения полярона в молекулярных полинуклеотидных цепочках конечной длины. // Математическая биология и биоинформатика. 2016. Т. 11. Вып. 2. С. 141-158. https://doi.org/10.17537/2016.11.141

22. Кориунова А.Н., Лахно В.Д. Особенности движения полярона в молекулярных полинуклеотидных цепочках конечной длины при наличии в цепочке локализованных возбуждений. // Математическая биология и биоинформатика. 2017. Т. 12. Вып. 1. С. 204-223. https://doi.org/10.17537/2017.12.204

23. Voityuk A.A., Rösch N., Bixon M., Jortner J. Electronic Coupling for Charge Transfer and Transport in DNA. // J. Phys. Chem. B. 2000. V. 104. Issue. 41. P. 9740-9745. https://doi.org/10.1021/jp001109w

24. Jortner J., Bixon M., Voityuk A.A., Rösch N. Superexchange Mediated Charge Hopping in DNA. // J. Phys. Chem. B. 2002. V. 106. P. 7599-7606. https://doi.org/10.1021/jp014232b

25. Lakhno V.D., Korshunova A.N. Formation of stationary electronic states in finite homogeneous molecular chains. // Mathematical biology and bioinformatics. 2010. Vol. 5. I. 1. P. 1-29. https://doi.org/10.17537/2010.5.1 
26. Lakhno V.D., Korshunova A.N. Bloch oscillations of a soliton in a molecular chain. // Eur. Phys. J. B. 2007. Vol. 55. P. 85-88. https://doi.org/10.1140/epjb/e2007-00045-3

27. Díaz E., Lima R. P. A., and Domínguez-Adame F. Bloch-like oscillations in the Peyrard-Bishop-Holstein model. // Phys. Rev. B. 2008. Vol. 78. Issue 13. P. 134303.5 p. https://doi.org/10.1103/PhysRevB.78.134303

28. Korshunova A.N., Lakhno V.D. A new type of localized fast moving electronic excitations in molecular chains. // Physica E. 2014. V. 60. P. 206209. https://doi.org/10.1016/j.physe.2014.02.025

\section{Оглавление}

1 Введение . . . . . . . . . . . . . . . . . . . . 3

2 Динамическая модель дискретной холстейновской цепочки . 3

3 Равномерное движения заряда . . . . . . . . . . . . . . . . . 4

4 Неравномерное движения заряда. . . . . . . . . . . . . . . . 9

5 Выводы ...................... 14

Библиографический список . . . . . . . . . . . . . . . 15 